\title{
Predação e canibalismo entre protozoários ciliados (Ciliophora: Entodiniomorphida: Ophryoscolecidae) no rúmen de ovinos (Ovis aries)
}

\author{
Isabel Martinele \& Marta D’Agosto
}

Departamento de Zoologia, Instituto de Ciências Biológicas, Universidade Federal de Juiz de Fora. Campus Universitário, 36036-900 Juiz de Fora, Minas Gerais, Brasil. E-mail: isabelmartinele@yahoo.com.br; marta.dagosto@ufjf.edu.br

\begin{abstract}
Predation and cannibalism among ciliate protozoans (Ciliophora: Entodiniomorphida: Ophryoscolecidae) in the rumen of sheep (Ovis aries). The objective of the present study was to record the occurrence of predation and cannibalism among ciliate protozoans in the rumen of sheep. The analyzed samples consisted of ruminal contents of five fistulated Santa Inez crossbred sheep, maintained in a natural caatinga (scrubland) pasture in the municipality of Sertânia, Pernambuco. Six samples of ruminal content were obtained from each sheep (total of 30 samples) and fixed in formalin at $18.5 \%$ (v/v). One $1 \mathrm{ml}$ aliquot was obtained from each sample of ruminal content and received tree drops of a lugol solution for staining and identification of protozoa ciliates. These aliquots were analyzed in slides and coverslips, at randomly determined fields. An instance of predator-prey interaction was observed among ciliates of Ophryoscolecidae Stein, 1859, where Elytroplastron bubali (Dogiel, 1928) preyed on Enoploplastron triloricatum (Dogiel, 1925) and on species of Epidinium Crawley, 1923 and Entodinium Stein, 1859. Cases of cannibalism were observed among specimens of $E$. bubali, which consist of the first record this type of interaction in this species. This is, moreover, the first record of occurrence of $E$. bubali in the rumen of sheep in Brazil.
\end{abstract}

KEY WORDS. Caatinga; competition; entodiniomorphs; predator; prey.

RESUMO. O objetivo deste trabalho foi registrar ocorrências de predação e canibalismo em protozoários ciliados no rúmen de ovinos. Foram analisadas amostras de conteúdo ruminal de cinco ovinos mestiços Santa Inês, fistulados no rúmen, mantidos em pastagem natural de caatinga, no município de Sertânia, Pernambuco. Foram obtidas seis amostras de conteúdo ruminal de cada ovino, totalizando 30 amostras, as quais foram fixadas em formalina $18,5 \%(\mathrm{v} / \mathrm{v})$. De cada amostra foi retirada uma alíquota de $1 \mathrm{ml}$ de conteúdo ruminal ao qual se adicionaram três gotas de solução de lugol para a coloração e identificação dos protozoários ciliados. Estas alíquotas foram analisadas utilizando-se lâminas e lamínulas, em campos determinados randomicamente. Constatou-se a ocorrência de interações do tipo predador-presa entre ciliados de Ophryoscolecidae Stein, 1859, onde Elytroplastron bubali (Dogiel, 1928) apresentou comportamento predatório sobre Enoploplastron triloricatum (Dogiel, 1925) e sobre outras espécies de Epidinium Crawley, 1923 e de Entodinium Stein, 1859. Foram ainda observados casos de canibalismo entre espécimes de $E$. bubali, o que consiste no primeiro registro deste tipo de interação nesta espécie. Registra-se, ainda, pela primeira vez a ocorrência de E. bubali no rúmen de ovinos no Brasil.

PALAVRAS-CHAVE. Caatinga; competição; entodiniomorfos; predador; presa.

Os protozoários ciliados do rúmen (Ciliophora, Entodiniomorphida, Ophryoscolecidae) são endosimbiontes de herbívoros ruminantes e compõem populações que habitam também o retículo e o omaso (SÁlvio \& D'Agosto 2001), além de outros compartimentos gástricos de herbívoros não ruminantes (Dehority 1987). Esta comunidade que habita o rúmen é constituída ainda por bactérias e fungos, os quais, juntamente com os protozoários ciliados, estabelecem uma simbiose mutualística com o hospedeiro. Entretanto, quando consideradas as interações intra-específicas e interespecíficas dentro desta comunidade, estas podem ser sinergéticas, estabelecendo complexas relações de interdependência; ou antagônicas, podendo resultar em mecanismos de predação e canibalismo (DеноRITy 1998).

Apesar destas interações serem determinantes para a dinâmica e a composição das populações que constituem a comunidade ruminal, pouco se conhece sobre elas, principalmente quando consideradas apenas as interações estabelecidas entre os protozoários ciliados.

LUBINSKY (1957) registrou casos de predação entre determinadas espécies de ciliados pertencentes à Ophryoscolecidae Stein, 1859, Diplodiniinae Lubinsky, 1957, os quais considerou como sendo acidentais, ocorrendo comumente nas espéci- 
es de maior tamanho corporal. Neste caso, as presas consistiram predominantemente de espécies sem espinhos, visto que a esta estrutura é atribuída a função de defesa.

Estudos acerca da dinâmica populacional de protozoários ciliados no rúmen demonstraram que alguns grandes entodiniomorfidas não estabelecem populações mistas, o que ocorre devido a uma clara relação de antagonismo entre estas populações (EAdie 1962a,b, Imai et al. 1979). Segundo EAdie (1967), o antagonismo entre espécies de protozoários ciliados, pode acarretar predação interespecífica. Um exemplo conhecido deste tipo de interação é a atividade predatória de Polyplastron multivesiculatum (Dogiel \& Fedorowa, 1925) sobre espécies de Epidinium Crawley, 1923 e de Eudiplodinium maggii (Fiorentini, 1889), sendo que nestes casos a predação é claramente não acidental, visto que pode levar à completa remoção das populações que constituem as presas (EAdie 1962a,b, 1967, ImAi et al. 1979).

Williams \& Coleman (1992) apresentaram uma relação de exemplos de predação entre protozoários ciliados do rúmen. Dentre estes relatos, esta predação foi estudada detalhadamente apenas entre as espécies $P$. multivesiculatum, a qual apresenta intensa atividade predatória sobre Epidinium sp.; e Entodinium bursa Stein, 1858, que após engolfar e digerir E. caudatum Stein, 1858, incorpora aminoácidos, purinas, proteínas e ácidos nucléicos utilizando-os como nutrientes.

Relatos de canibalismo entre protozoários ciliados no rúmen são ainda mais escassos que os de predação, sendo conhecido entre indivíduos de P. multivesiculatum (EADIE 1967). Nestes casos, a predação pode ocasionar alterações na densidade e na composição das populações de protozoários, variações no tamanho celular e no tempo de geração de novos organismos, além do desenvolvimento de polimorfismo, especialmente nas espécies que se constituem em presas (EADIE 1962a,b, 1967, Williams \& Coleman 1992).

Considerando a escassez de informações sobre as relações estabelecidas entre as populações de protozoários ciliados no rúmen, objetivou-se com o presente estudo investigar evidências do comportamento alimentar de E. bubali. Este artigo apresenta novos registros de predação e canibalismo entre protozoários ciliados no rúmen de ovinos mestiços Santa Inês mantidos em pastagens naturais de caatinga, Pernambuco, Brasil.

\section{MATERIAL E MÉTODOS}

O experimento foi desenvolvido no Centro de Treinamento em Caprino-Ovinocultura pertencente à Empresa Pernambucana de Pesquisa Agropecuária, Sertânia, Pernambuco. As coletas foram realizadas em julho de 2005 e janeiro de 2006. A análise laboratorial foi desenvolvida no Laboratório de Protozoologia do Programa de Pós-Graduação em Ciências Biológicas, Comportamento e Biologia Animal, Universidade Federal de Juiz de Fora, Minas Gerais.

Foram utilizados cinco ovinos mestiços Santa Inês, machos, castrados, com idade entre 10-12 meses, fistulados no rúmen e mantidos em criação extensiva em pastagem de caatinga, onde permaneceram de setembro de 2004 a janeiro de 2006. Os animais não receberam nenhum tipo de suplementação, sendo a dieta composta exclusivamente pela vegetação disponível numa pastagem de caatinga, que compreendeu uma área de 37 ha $\left(8^{\circ} 03^{\prime} 38^{\prime \prime}\right.$ S e $\left.37^{\circ} 13^{\prime} 32^{\prime \prime} \mathrm{W}\right)$. Além da pastagem, os animais receberam apenas sal mineral e água ad libitum. Os ovinos tinham acesso à pastagem durante o período das 7:00 às 17:00 h e eram recolhidos ao aprisco após as 17:00 $\mathrm{h}$ para $\mathrm{o}$ período de pernoite. O procedimento descrito foi adotado durante todo o período experimental de forma que não se limitasse aos meses de coleta.

Determinou-se o máximo de duas coletas de conteúdo ruminal por dia de forma a atenuar os efeitos sobre o tempo de forrageamento dos animais, visto que estes eram trazidos ao aprisco para a realização da coleta. Os horários das coletas seguiram o tempo de soltura dos animais, sendo a primeira realizada antes de se soltar os animais (zero hora) e as demais as duas, quatro, seis, oito e dez horas após o animal iniciar o pastejo. Coletaram-se amostras de conteúdo ruminal para cada animal em cada um dos horários de amostragem, perfazendo um total de 30 amostras coletadas durante o mês de julho de 2005, e 24 amostras em janeiro de 2006. O menor número de amostras obtidas na estação seca ocorreu em virtude da morte de um dos animais do grupo experimental durante a segunda fase de coleta, por picada de serpente. Cada amostra consistiu de $20 \mathrm{ml}$ de conteúdo ruminal, sendo estas imediatamente fixadas em formol na proporção de 1:2 (Dehority 1984) e armazenadas em frascos plásticos devidamente etiquetados.

A quantificação dos espécimes de Elytroplastron bubali foi efetuada em câmara Sedgewick-Rafter, segundo DeнoRiтy (1984), sendo que de cada amostra homogeneizada foi pipetado $1 \mathrm{ml}$ de conteúdo e transferido para tubos de ensaio, onde foram acrescentadas três gotas de lugol, em substituição ao verde brilhante conforme com a modificação proposta por D'Agosto \& CARneiro (1999). Após 15 minutos o conteúdo recebia a adição de $9 \mathrm{ml}$ de glicerina a 30\%. Para proceder à quantificação, de cada tubo de ensaio, foi pipetado $1 \mathrm{ml}$ do conteúdo para preencher a câmara de Sedgewick-Rafter. Utilizando-se uma grade de contagem em uma das oculares, foram quantificados os ciliados presentes em 50 campos e posteriormente, após rotação da câmara em $180^{\circ}$, mais 50 campos. O cálculo do número total de ciliados por mililitro de conteúdo foi feito multiplicando-se os valores encontrados por 80 e por 20. Tais valores correspondem à superfície total da câmara de contagem e à diluição (Dehority 1984). A quantificação dos protozoários ciliados em processo de reprodução seguiu a técnica descrita anteriormente. A identificação dos ciliados baseou-se em Ogimoto \& Imai (1981).

Os comportamentos de predação e canibalismo foram registrados conforme a técnica de observação de amostragem instantânea (AlTMANN 1974), adaptada ao estudo de protozoários (D'Agosto et al. 2003), sendo este registro feito por meio de 
fotomicrografias digitais. Para este procedimento, utilizou-se uma alíquota de $1 \mathrm{ml}$ de conteúdo ruminal de cada amostra coletada, ao qual foram acrescentadas três gotas de lugol de forma a permitir a visualização das placas esqueléticas para posterior identificação. Cada uma destas alíquotas foi colocada entre lâmina e lamínula e analisadas em microscópio fotônico (Olympus BX 41), em campos determinados randomicamente.

O número de ciliados E. bubali foi comparado entre os horários de amostragem, em ambas estações, utilizando-se de análise de variância (Anova) seguida pelo teste de NewmanKeuls ( $\mathrm{p}<0,05)$. O teste $\mathrm{t}$ de Student foi utilizado para comparar o número de ciliados em cada horário de amostragem entre as estações chuvosa e seca. O programa estatístico utilizado foi o Bioest 2.0 (Ayres et al. 2000).

Os procedimentos que envolvem os ovinos foram analisados e aprovados pelo Comitê de Ética do Departamento de Zootecnia da Universidade Federal Rural de Pernambuco.

\section{RESULTADOS E DISCUSSÃO}

Não houve diferença significativa no número destes ciliados quando comparados entre os horários de amostragem em cada uma das estações ( $p$ > 0,05) (Tab. I). Também não foi constatada diferença $(p>0,05)$ quando o número de ciliados em cada um dos horários de amostragem foi comparado entre as estações. Estas variações ao longo do dia tendem a ser reduzidas quando os animais estão em pastejo, sem horário fixo de alimentação.

Tabela I. Concentração média de organismos de $E$. bubali por mililitro de conteúdo ruminal $\left(\times 10^{4}\right)$ em ovinos mestiços Santa Inês em diferentes horários de amostragem e estações, em pasta-gem natural de caatinga, Pernambuco.

\begin{tabular}{ccc}
\hline Horários de amostragem $^{*}$ & Estação chuvosa & Estação seca \\
\hline 0 & $1,72 \pm 1,64$ & $1,52 \pm 1,51$ \\
2 & $1,12 \pm 0,74$ & $1,04 \pm 1,28$ \\
4 & $1,02 \pm 0,73$ & $0,96 \pm 0,87$ \\
6 & $2,30 \pm 2,20$ & $0,76 \pm 0,74$ \\
8 & $0,41 \pm 0,46$ & $1,24 \pm 0,57$ \\
10 & $0,92 \pm 0,93$ & $1,60 \pm 0,87$ \\
\hline Média & $1,25 \pm 1,31$ & $1,18 \pm 0,96$ \\
\hline
\end{tabular}

* Antes dos animais serem soltos ao pasto (zero hora) e após 2, 4, 6,8 e 10 horas de pastejo.

Segundo Warner (1962), em animais com acesso livre ao alimento ou pastagens, as divisões celulares dos ciliados ocorreriam ao acaso durante todo o dia. Possivelmente esta aleatoriedade leva à estabilização das formas em divisão ao longo do dia, o que contribui para a manutenção da densidade das populações destes protozoários ao longo do dia, no ambiente ruminal.
Embora E. bubali tenha ocorrido em baixas concentrações (Tab. I), esta espécie apresentou durante as estações chuvosa e seca, respectivamente, 80 e 100\% de prevalência. Estes dados se destacam porque os poucos registros da ocorrência desta espécie no Brasil, foram no rúmen de bovinos (Dehority 1986, D’Agosto \& Guedes 2000, D'Agosto et al. 2001) e de bubalinos (Franzolin Neto et al. 1991). Portanto, este é o primeiro registro da ocorrência desta espécie no rúmen de ovinos no Brasil.

Foram registrados casos de predação e canibalismo entre protozoários ciliados Ophryoscolecidae, com a observação do comportamento predatório de E. bubali (Fig. 1) sobre Enoploplastron triloricatum (Dogiel, 1925), (Fig. 2); e sobre espécies de Entodinium Stein, 1859 (Fig. 3) e de Epidinium (Figs 4-6). Constatou-se ainda a ocorrência de canibalismo entre espécimes de E. bubali (Figs 7-9). Em alguns destes espécimes foi possível, ainda, a observação de fases iniciais da fissão binária transversal homotetogênica (Figs 2, 3, 5 e 7-9).

A predação de Enoploplastron triloricatum por E. bubali foi observada em diferentes amostras com relativa freqüência. A predação interespecífica entre protozoários ciliados do rúmen foi considerada por LuBINSKY (1957) como sendo acidental, quando registrou a atividade predatória de E. bubali sobre espécies de Entodinium e de Metadinium Awerinzew \& Mutafowa, 1914 e de E. maggii sobre Entodinium sp. Entretanto, EADIE (1962a, b, 1967) demonstrou que este comportamento tem bases mais complexas, as quais são atribuídas a relações de competição, geralmente por alimento, o que gera atividades de predação e canibalismo. Além disso, certas espécies de protozoários ciliados se constituem em importantes fontes de nutrientes e energia para outras espécies. Provavelmente, por isso se observou em grande parte dos registros, que os espécimes de E. bubali se encontravam em fase inicial de reprodução, período este que demanda um significativo gasto energético.

A literatura relata a existência de canibalismo entre organismos da espécie P. multivesiculatum (EADIE 1967). Tal comportamento pode decorrer de mecanismos de competição intraespecífica ou quando outras fontes de alimento encontrem-se em escassez, como por exemplo, quando o hospedeiro passa por um período prolongado de restrição alimentar (EADIE 1967). No presente estudo, o canibalismo foi registrado, pela primeira vez, entre indivíduos da espécie E. bubali.

As interações ecológicas entre os protozoários ciliados em ruminantes são pouco elucidadas, sendo limitados os registros de predação e canibalismo. Estas interações são capazes de modificar a composição da comunidade ruminal (EADIE 1962a, b, 1967, Imai et al. 1979), portanto, a observação destes fatores pode pontuar questões que ajudem a esclarecer a dinâmica destes organismos no ambiente ruminal e seu papel no metabolismo do hospedeiro.

A escassez de estudos desta natureza se deve à dificuldade em manter estes organismos in vitro. Um meio alternativo para estudos sobre o comportamento de protozoários ciliados 


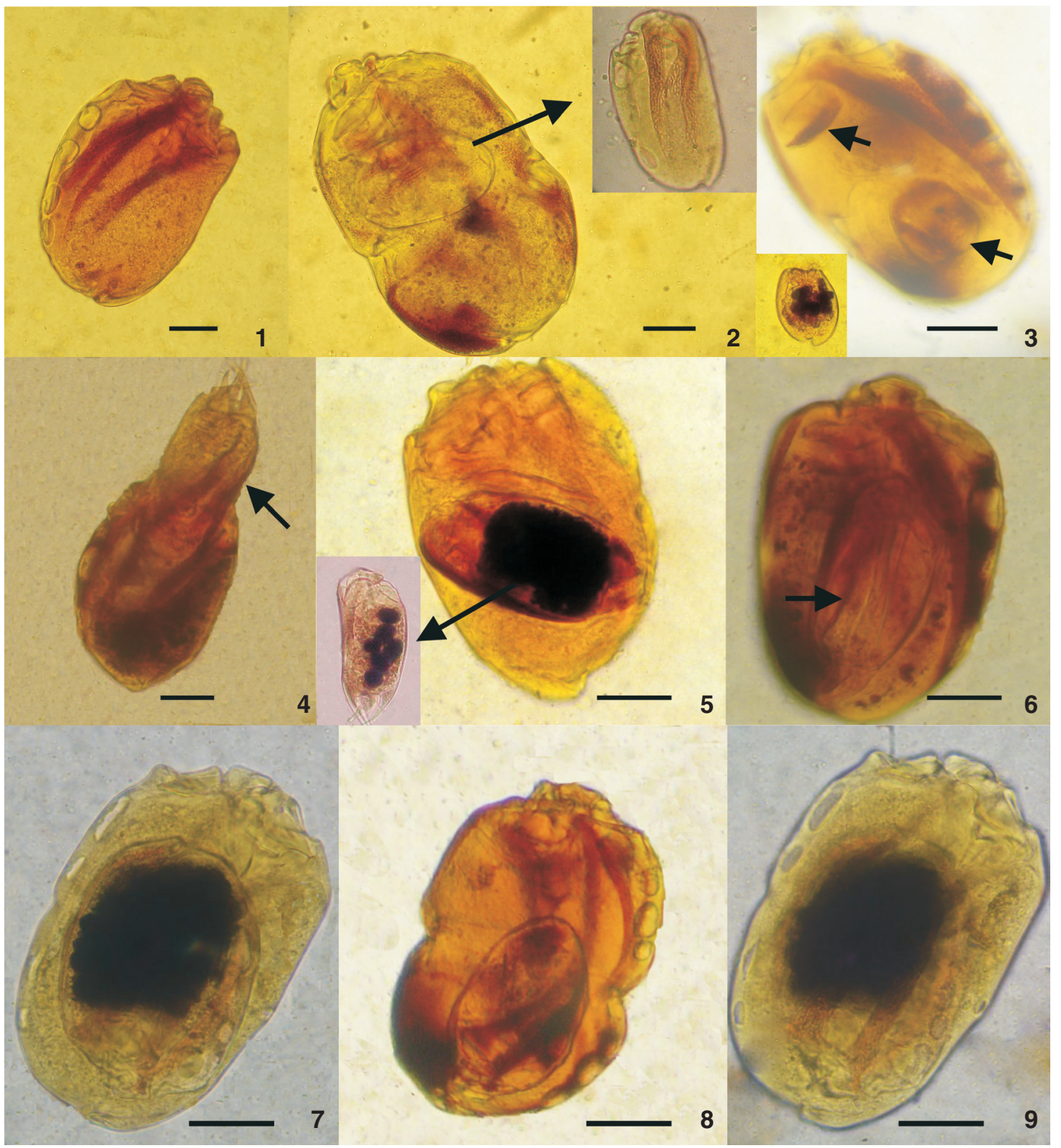

Figuras 1-9. Fotomicrografias evidenciando os comportamentos de predação e canibalismo em protozoários ciliados do rúmen de ovinos. (1) Elytroplastron bubali; após predar: (2) Enoploplastron triloricatum; (3) Entodinium sp.; (4-6) Epidinium sp.; (7-9) canibalismo entre indivíduos da espécie $E$. bubali. As setas menores indicam fases iniciais de fissão binária transversal homotetogênica. Barra = $10 \mu m$.

no rúmen é a utilização de amostras de conteúdo ruminal fixadas em formol na proporção de 1:2. Desta forma, é possível obter registros através da observação de amostragem instantânea (Altmann 1974) de forma adaptada ao estudo destes protozoários (D'Agosto et al. 2003) que permite a inferência de questões relativas ao comportamento destes organismos no ambiente ruminal.

\section{AGRADECIMENTOS}

À Coordenação de Aperfeiçoamento de Pessoal de Nível Superior pela concessão de bolsa de mestrado a Isabel Martinele. À Empresa Pernambucana de Pesquisa Agropecuária do estado de Pernambuco, pela cessão da área experimental e manutenção dos animais. À FAPEMIG pelo auxílio à publicação dos resultados.

Revista Brasileira de Zoologia 25 (3): 451-455, September, 2008 


\section{LITERATURA CITADA}

Altmann, J. 1974. Observational study of behaviour: Sampling methods. Behaviour 48: 227-265.

Ayres, M.; M.J.R. Ayres; D.L. Ayres; A.S. Santos. 2000. BioEstat 2.0: aplicações estatísticas nas áreas de ciências biológicas e médicas. Brasília, Sociedade Civil Mamirauá, CNPq.

D'Agosto, M. \& M.E. Carneiro. 1999. Evaluation of lugol solution used for counting rumen ciliates. Revista Brasileira de Zoologia 16: 725-729.

D’Agosto, M. \& P.M.M. Guedes. 2000. Caracterização das populações de ciliados (Protista, Ciliophora) do rúmen de bovinos de corte no estado de Minas Gerais, Brasil. Revista Brasileira de Zoociências 2 (1): 81-90.

D’Agosto, M.; I.C.V. Siqueira \& N.B. Espírito-Santo. 2001. Comportamento e distribuição de protozoários ciliados (Protista, Ciliophora) no rúmen e no retículo de bovinos submetidos ao jejum. Revista Brasileira de Ciência Veterinária 8 (1): 16-18.

D’Agosto, M.T.; H.H. Santos-Prezoto \& R.J.P. Dias. 2003. Estudando o comportamento de protozoários, p. 22-28. In: K. Delclaro \& F. Prezoto (Eds). As distintas faces do comportamento animal. São Paulo, Conceito, 276p.

Dehority, B.A. 1984. Evaluation of subsampling and fixation procedures used for counting rumen Protozoa. Applied Environmental Microbiology 48: 182-185.

Dehority, B.A. 1987. Rumen Ophryoscolecid Protozoa in the hindgut of the Capybara (Hydrochoerus hydrochaeris). Journal of Parasitology 34 (2): 143-145.

Dehority, B.A. 1986. Rumen ciliate fauna of some brazilian cattle: Occurrence of several ciliates new to the rumen, including the cycloposthid Parentodinium africanum. Journal of Protozoology 33: 416-421.
Dehority, B.A. 1998. Microbial interactions in the rumen. Revista de La Facultad de Agronomía Luz 15: 69-86.

EADIE, M. 1962a. The development of rumen microbial populations in lambs and calves under various conditions of management. Journal General Microbiology 29: 563578.

EADIE, M. 1962b. Inter-relationships between certain rumen ciliate protozoa. Journal General Microbiology 49: 175194.

EAdIE, M. 1967. Studies on the ecology of certain rumen ciliate protozoa. Journal General Microbiology 49: 175-194.

Franzolin Neto, R.; J.C.M. Nogueira Filho \& M.E.M. Oliveira. 1991. Efeitos de dietas com diferentes níveis de proteína sobre os protozoários ciliados no rúmen de búfalos (Bubalus bubalis L.). Pesquisa Agropecuária Brasileira 26 (4): 487493.

Imai, S.; M. Katsuno \& K. Ogimoto. 1979. Type of the pattern of the rumen ciliate composition of the domestic ruminants and the predator-prey interaction of the ciliates. Japanese Journal Zootechny Science 50 (2): 79-87.

Lubinsky, G. 1957. Note on the philogenetic significance of predatory habits in the Ophryoscolecidae (Ciliata: Oligotricha). Canadian Journal Zoology 35: 579-580.

Ogimoto, K. \& S. Imai. 1981. Atlas of Rumen Microbiology. Tokyo, Japan Scientific Societies Press, VIII+231p.

SÁlvio, G.M.M \& M. D’Agosto. 2001. Ciliados nas cavidades do estômago de bovinos. Arquivo Brasileiro de Medicina Veterinária e Zootecnia 53 (6): 686-690.

WARner, A.C.I. 1962. Some factors influencing the rumen microbial population. Journal General Microbiology 28: 129-146.

Williams, A.G. \& G.S. Coleman. 1992. The rumen protozoa. New York, Springer-Verlag, 423p.

Submitted: 20.IX.2007; Accepted: 01.IX.2008.

Editorial responsibility: Walter A.P. Boeger 